\title{
When "no" might not quite mean "no"; the importance of informed and meaningful non-consent: results from a survey of individuals refusing participation in a health-related research project Brian Williams*1, Linda Irvine ${ }^{1}$, Alison R McGinnis ${ }^{1}$, Marion ET McMurdo ${ }^{2}$ and Iain K Crombie ${ }^{1}$
}

Address: ${ }^{1}$ Section of Public Health, Division of Community Health Sciences, Mackenzie Building, Kirsty Semple Way, University of Dundee, Dundee, UK and 2Division of Medicine \& Therapeutics, Ninewells Hospital \& Medical School, University of Dundee, Dundee, UK

Email: Brian Williams* - b.y.williams@chs.dundee.ac.uk; Linda Irvine - m.a.j.irvine@chs.dundee.ac.uk; Alison R McGinnis - alison.mcginnis@tuht.scot.nhs.uk; Marion ET McMurdo - m.e.t.mcmurdo@dundee.ac.uk; Iain K Crombie - i.k.crombie@chs.dundee.ac.uk

* Corresponding author

Published: 26 April 2007

BMC Health Services Research 2007, 7:59 doi:10.1 186/1472-6963-7-59

This article is available from: http://www.biomedcentral.com/1472-6963/7/59

(c) 2007 Williams et al; licensee BioMed Central Ltd.

This is an Open Access article distributed under the terms of the Creative Commons Attribution License (http://creativecommons.org/licenses/by/2.0), which permits unrestricted use, distribution, and reproduction in any medium, provided the original work is properly cited.
Received: 4 September 2006

Accepted: 26 April 2007

\begin{abstract}
Background: Low participation rates can lead to sampling bias, delays in completion and increased costs. Strategies to improve participation rates should address reasons for non-participation. However, most empirical research has focused on participants' motives rather than the reasons why non-participants refuse to take part. In this study we investigated the reasons why older people choose not to participate in a research project.
\end{abstract}

Methods: Follow-up study of people living in Tayside, Scotland who had opted-out of a cross-sectional survey on activities in retirement. Eight hundred and eighty seven people aged 65-84 years were invited to take part in a homebased cross-sectional survey. Of these, 47I refused to take part. Permission was obtained to follow-up 417 of the refusers. Demographic characteristics of people who refused to take part and the reasons they gave for not taking part were collected.

Results: $54 \%$ of those invited to take part in the original cross-sectional survey refused to do so. However, $61 \%$ of these individuals went on to participate in the follow-up study and provided reasons for their original refusal. For the vast majority of people initial non-participation did not reflect an objection to participating in research in principle but frequently stemmed from barriers or misunderstandings about the nature or process of the project itself. Only $28 \%$ indicated that they were "not interested in research". The meaningfulness of expressions of non-consent may therefore be called into question. Hierarchical log-linear modelling showed that refusal was independently influenced by age, gender and social class. However, this response pattern was different for the follow-up study in which reasons for nonparticipation in the first survey were sought. This difference in pattern and response rates supports the likely importance of recruitment issues that are research and context specific.

Conclusion: An expression of non-consent does not necessarily mean that a fully informed evaluation of the pros and cons of participation and non-participation has taken place. The meaningfulness of expressions of non-consent may therefore be a cause for concern and should be subject to further research. Many reasons for non-participation may be specific to a particular research topic or population. Information sheets should reflect this by going beyond standardised guidelines for their design and instead proactively seek out and address areas of concern or potential misunderstanding. The use of established behavioural theory in their design could also be considered. 


\section{Background}

Recent years have seen changes to consent procedures, data access, and research governance in the UK, all of which are designed to ensure that health research is conducted ethically and with integrity. The motivation for these changes are rooted in an increasing culture of accountability of professionals and a desire to address growing public and media concern about the ethical conduct of research. However, some of these developments may threaten the delivery of valid and reliable research $[1,2]$. At the centre of these challenges is the ongoing struggle to achieve high recruitment/response rates in community based research projects.

Low response and participation rates undermine statistical power and increase the probability of bias. Nonresponders have, for example, been found to be more likely to be smokers, to have poorer health and to make less use of health care services [3-5]. Furthermore, these problems are exacerbated among particular social groups: response rates usually fall with age [6-8] and are lower in those with less education and poorer socio-economic status $[9,10]$. While knowing the magnitude of these differences may enable researchers to estimate or to adjust for the bias introduced by non-response $[3,5,7]$, this is second best to a policy of prevention or reduction of initial non-response.

Numerous attempts have been made to identify effective strategies to maximise participation in both experimental and observational studies [11-13]. While a recent Cochrane review that identified 372 randomised controlled trials of interventions aimed to increase response rates to postal questionnaires identified a range of effective strategies [14] (envelope size, paper colour, financial incentives and ease of opt out) meta-analyses frequently show significant levels of heterogeneity between studies [15]. This indicates that strategies that may be highly effective for one social group in one setting may be less effective or even counterproductive in another. While this suggests that the reasons behind non-response or poor recruitment are likely to be variable, interventions to improve recruitment have seldom been based on the findings of exploratory studies of the reasons for non-response or recruitment in the first place. Consequently, even interventions that have some degree of demonstrable effectiveness are unlikely to be able to address issues that stem from the unique and idiosyncratic nature and context of individual projects where potential respondents may face specific practical problems or concerns.

Even where effectiveness is demonstrated questions remain. The finding that a particular strategy improves recruitment or response does not mean that it has either addressed the underlying concerns or practical problems associated with low rates. Instead it may simply be masking the problem. For example, monetary and non-monetary incentives may encourage people to participate but do nothing to alleviate their concerns about how the data are used or whether it will be treated confidentially.

Numerous studies have examined the motives and experiences of individuals who participate in research [16-21]. However, few have explored reasons for non-participation. This is unsurprising given the potential ethical and practical difficulties of accessing individuals who have already said that they do not wish to participate in a piece of research. However, structural, ethical and procedural changes for research (the increasing requirement to seek consent even where there is no patient contact, to have a formal "opt-in" rather than an "opt-out" process, and the increasing inability of health researchers in the UK to recruit participants directly rather than through a clinical intermediary) all mean that response rates have increasing potential for bias [22,23]. Consequently, it is perhaps more important than ever to focus not only on what motivates response and participation but rather what nonresponse or refusal to participate actually means and what the reasons behind it are. In this paper we report the findings of a survey of the characteristics and reasons for nonresponse in a study of attitudes to physical activity among older people. We then go on to discuss in detail possible responses and considerations. We do not present solutions but simply intend to encourage researchers to look at the issue of research non-participation as a behaviour that can be explored, analysed, and theorised just as other behaviours are similarly studied in other contexts of health.

\section{Methods}

In 2002-4 we conducted a cross-sectional survey based on structured face to face interviews examining reasons for low physical activity among older people. These details, along with the findings of the study, have been reported elsewhere [24]. Subjects aged 65 to 84 years were identified from 16 GP practices in Dundee through age/gender registers. All were allocated a deprivation score [25]: scores of 1 and 2 were classed as areas of low deprivation; 3,4 and 5 as medium; and 6 and 7 as areas of high deprivation [26]. A stratified random sample (age in 10 year groups, gender and three deprivation groups) of 1064 subjects was drawn. GPs screened the lists of names and 100 were excluded (terminal illness, dementia, living in a nursing home). Twenty subjects could not be traced at the addresses given. A further 57 subjects were excluded at initial contact because they were unsuitable (e.g. admitted to hospital, language barrier, bereavement, extreme frailty). A total of 887 subjects suitable for inclusion were invited to take part in the study. 
Potential participants were initially contacted by a letter from their GP. This letter, and the information leaflet, stated that the study would investigate physical and social activities with the aim of improving facilities for older people. They were given the opportunity to opt out of the research by returning a postcard to the practice. If the postcard was not returned to the practice within three weeks, they were contacted by a research nurse to arrange an interview.

\section{Follow up of those who refused to be interviewed}

We obtained permission from our Local Research Ethics Committee (LREC) for GPs to approach people who refused to take part in the main survey. We were aware that the our LREC, along with many others in the UK, were increasingly requiring researchers to use an "opt-in" approach in which individuals are written to by their general practitioner and asked to respond if they would like to receive more information about the project (as opposed to an "opt-out" method in which non-response to such a communication would result in the researchers approaching them). For the main survey the LREC originally requested an opt-in approach. However, after the researchers highlighted the degree to which sampling bias might be created in using an opt-in approach, the LREC agreed to the use of an opt-out approach. Given the preference for an opt-in stance and its underlying rationale to minimise burden on potential participants we were aware that any subsequent request to the LREC to approach people who had already requested not to be involved by actively telephoning or returning a card would be contentious. We therefore requested and obtained permission to ask only a small number of essential questions. We also agreed to ensure that the time required to complete the questionnaire be short, and that our covering letter overtly stress that participation was voluntary and that we respected their decision not to take part. These requirements precluded the possibility of using a qualitative approach to data collection. A further reason for the method and its brevity was simply to encourage as high a response rate as possible as it was assumed that people who had refused to take part in the main study would not be greatly motivated.

GPs sent a short questionnaire to those individuals who refused to take part in order to ascertain their reasons for non-participation. They were invited to return the questionnaire to their GP in a stamped addressed envelope which was provided. Only one letter was sent in order to minimize the potential intrusiveness of the study.

\section{The follow-up questionnaire}

The follow-up questionnaire was designed to be as brief as possible in order to maximize likely response. Eight questions were selected by drawing on both the literature con- cerning non-response and poor recruitment, and the reasons expressed for non-response among those who had refused consent when the researcher had contacted them by telephone. The questionnaire included two questions on each of four topic areas: understandings about the research; concerns around privacy; personal reasons (e.g. health); lack of interest in research. A single open question was also asked in order to allow expression of other reasons not embodied in the previous eight.

\section{Analysis}

Chi-squared tests were performed to investigate univariate associates with refusal. Model fitting using hierarchical log-linear modelling was used to identify significant independent predictors [27].

\section{Results}

Of the 887 subjects who were invited to take part in the main survey, 384 refused by completing and returning a card indicating their unwillingness to take part. A further 91 refused when telephoned or when visited, and 3 gave no response, leaving 409 who were interviewed. It is noticeable that of those who refused, $80 \%$ did so by returning the postcard.

Refusal to take part in the main survey varied significantly by age, gender and level of deprivation (Table 1). When fitted into a hierarchical loglinear model each of the three terms showed a significant independent association with refusal. Refusal was higher in the older group, by female gender and increased with increasing level of deprivation. None of the higher order interactions were significant.

The 471 people who refused by card return or at personal contact were considered for the follow up study. After GP exclusions a total of 417 were approached. Overall $61 \%$ of those who refused to take part in the main survey returned a follow-up questionnaire giving their reasons for refusal. The effect of age, gender and deprivation on the return rate of the follow-up questionnaires was quite different to their effect on participation in the cross-sectional survey (Table 2). Thus the older group had a significantly lower non-participation rate than the younger group. Furthermore, there was little difference between men and women in non-response and the effects of deprivation were inconsistent and non-significant. No higher order interactions were significant.

The follow-up questionnaire comprised eight questions, which participants could answer yes, no, or give no response. By far the most commonly selected reason for not taking part in the main study was that the participants considered that they did not do enough exercise to be useful for research (Table 3). Only a minority (28\%) indicated that they "were not interested in research" and even 
Table I: Numbers of subjects refusing and agreeing to take part in the main survey interviews

\begin{tabular}{|c|c|c|c|}
\hline & Refused $\mathbf{N}$ & Interviewed $\mathbf{N}$ & \\
\hline \multicolumn{4}{|l|}{ Age } \\
\hline $65-74$ years $(n=476)$ & $234(49.2 \%)$ & $242(50.8 \%)$ & \\
\hline $75-84$ years $(n=4 I 2)$ & $244(59.2 \%)$ & $168(40.8 \%)$ & $\chi^{2}=8.8 \mathrm{I}, \mathrm{Idf}, \mathrm{p}<0.0 \mathrm{I}$ \\
\hline \multicolumn{4}{|l|}{ Gender } \\
\hline Male $(n=428)$ & $216(50.5 \%)$ & $212(49.5 \%)$ & \\
\hline Female $(n=459)$ & $262(57.1 \%)$ & $197(42.9 \%)$ & $\chi^{2}=3.90, I d f, p<0.05$ \\
\hline \multicolumn{4}{|l|}{ Deprivation Level } \\
\hline Low $(n=35 I)$ & $165(47.0 \%)$ & $186(53.0 \%)$ & \\
\hline Medium $(n=322)$ & $186(56.0 \%)$ & $146(44.0 \%)$ & \\
\hline High $(n=204)$ & $127(62.3 \%)$ & 77 (37.7\%) & $\chi^{2}=13.0,2 \mathrm{df}, \mathrm{p}<0.00 \mathrm{I}$ \\
\hline TOTAL & $478(53.9 \%)$ & $409(46.1 \%)$ & \\
\hline
\end{tabular}

these had actually taken part by replying. The other most common answers reflected concerns about loss of privacy, either through research staff visiting them or by being asked questions about personal matters. We examined the data to see if the reasons provided differed between age, gender and deprivation groups (Table 4). Two significant differences were found. Unsurprisingly, older people were more likely to report their age as a reason for non-participation ( $\mathrm{p}<0.0001)$. In addition men were more likely than women to indicate that they were not interested in research (33\% versus $22 \%$; $\mathrm{p}=0.045)$.

Participants were also invited to add their own comments at the end of the questionnaire. Two key themes appeared within the data. The most commonly cited reasons reflected the perceived difficulty of participating, rather than a lack of willingness. Many participants indicated that they had too many other commitments to be able to take part in research. These often involved family ties, but could involve other activities:
"My wife does not keep very well and I need to watch over her"

"Family commitments prevent me from attending, organised activities"

"My other commitments will prevent me from participating"

A second dominant theme, research misunderstanding, reflected confusion about the nature and purpose of the research itself. Responses suggested that many may have declined to take part in the initial survey either because they regarded themselves as already physically active and therefore not appropriate for inclusion, or because they thought that the researchers had contacted them because they were felt to be in need in some way.

"I am pleased as I am, but thank you all for your care and kindness"

Table 2: Numbers of subjects who replied to the follow-up study

\begin{tabular}{|c|c|c|c|}
\hline & Replied N & No Reply N & \\
\hline \multicolumn{4}{|l|}{ Age } \\
\hline $65-74$ years $(n=205)$ & $116(56.6 \%)$ & $89(43.4 \%)$ & \\
\hline $75-84$ years $(n=212)$ & 140 (66.0\%) & $72(34.0 \%)$ & $\chi^{2}=3.93, I d f, p<0.05$ \\
\hline \multicolumn{4}{|l|}{ Gender } \\
\hline Male $(n=186)$ & 118 (63.4\%) & $68(36.6 \%)$ & \\
\hline Female $(n=231)$ & $138(59.7 \%)$ & $93(40.3 \%)$ & $\chi^{2}=0.6, I d f, p=0.44$ \\
\hline \multicolumn{4}{|l|}{ Deprivation Level } \\
\hline Low $(n=148)$ & $93(62.8 \%)$ & 55 (37.2\%) & \\
\hline Medium $(n=160)$ & $90(56.3 \%)$ & $70(43.7 \%)$ & \\
\hline High $(n=109)$ & 73 (70.0\%) & $36(30.0 \%)$ & $\chi^{2}=3.35,2 d f, p=0.19$ \\
\hline Total & $256(61.4 \%)$ & $161(38.6 \%)$ & \\
\hline
\end{tabular}


Table 3: Reasons for not taking part in the interview study $(\mathbf{N}=\mathbf{2 5 6})$

Yes (\%) No (\%) No box selected (\%)

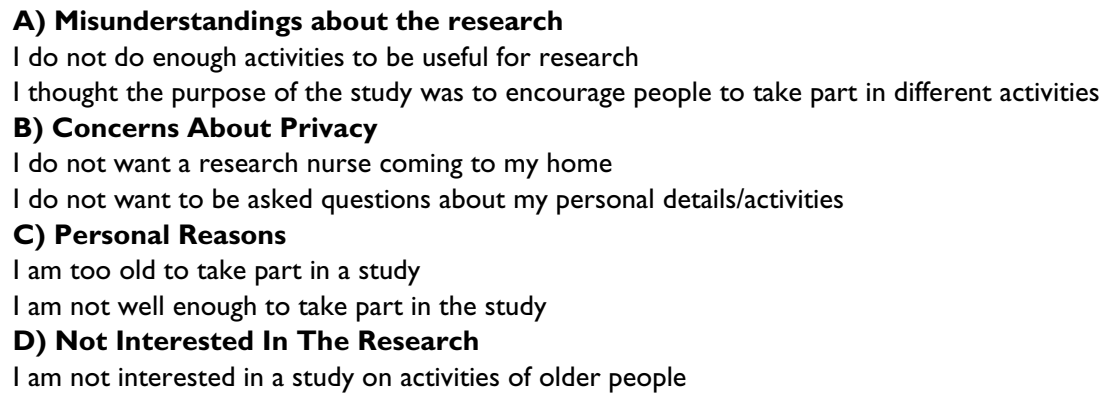

$\begin{array}{lll}55.5 & 18.1 & 26.4 \\ 32.3 & 26.0 & 41.7 \\ & & \\ 44.9 & 24.0 & 31.1 \\ 39.4 & 28.7 & 31.9 \\ & & \\ 37.4 & 30.3 & 32.3 \\ 28.0 & 39.0 & 33.1 \\ & & \\ 31.5 & 33.5 & 35.0 \\ 27.4 & 35.6 & 37.0\end{array}$

"I keep active in my own way, a little gardening etc, when necessary, at my own pace"

"My personal activities at present fulfil my needs"

"I'll no doubt soon be donning the old wooden overcoat"

\section{Discussion}

The study found that for the vast majority of people refusal to participate in the main survey did not reflect an objection to participating in research in principle but frequently stemmed from barriers or misunderstandings about the nature or process of the project itself. The meaningfulness of expressions of non-consent may therefore be called into question.

Response rates for the main survey varied by age, gender and social deprivation. However, this response pattern was different for the follow-up study in which reasons for non-participation were sought. This supports the likely importance of recruitment issues that are research and context specific, as opposed to generic issues alone.

This study is one of a very few that has accessed the views of individuals who have refused to take part in a research project. The scale and nature of data collection was constrained by sensitivity to the fact that participants had already refused to take part in our main survey. As a result there are a number of caveats that must be considered prior to discussion of the study findings. Firstly, it is possible that reasons given for non-participation may not reflect those truly responsible. Both social desirability (e.g. unwilling to admit to apathy) and recall may mean some reasons have been omitted. Secondly, the data relates only to older people. While some of the reasons for non-participation may apply to younger groups we do not have the data to support this. Thirdly, it is possible that despite assurances in regard to confidentiality the requirement that the follow-up questionnaire should be sent by their GP may have resulted in some people feeling intimidated and therefore either not responding at all (potentially resulting in some sampling bias) or by responding but providing "acceptable" rather than accurate reasons for original non-participation. Finally, the reasons for non-participation provided are in relation to a request for a detailed, structured interview in the home setting and may not apply to other data collection methods.

Despite these caveats our findings may be useful and informative for researchers in a number of ways. Firstly, they provide detail on likely response rates among older people and the tailoring of information sheets. Secondly, they raise questions over the meaningfulness not only of expressions of non-consent but also consent. Finally, they have implications not only for the design but also the recruitment procedure itself. These are dealt with in turn below.

\section{Response, refusal and information provision among older people}

Fewer than $50 \%$ of people invited to take part in a structured interview about social and physical activities agreed to take part. Review papers have reported that many studies among older people have lower response rates $[3,6,9,28]$. As in this study, others have reported that response rates usually fall with age [6-8] and are lower in those with poorer socio-economic status $[9,10]$. These variations suggest that the reasons for non-participation may be different between social groups and/or that the strength of such reasons might differ. Consequently, information sheets or recruitment strategies that are based on a "one size fits all" basis may at best be inefficient and at worst

inappropriate.

Within the UK information sheets are increasingly being standardised under COREC (Central Office for Research Ethics Committees) requirements. However, their design is rarely empirically based and almost always atheoretical 
Table 4: Reasons for Refusal To Participate By Age, Gender and Deprivation

\section{A) Misunderstandings about the research}

I do not do enough activities to be useful for research

$65-74$ years

$75-84$ years

Male

Female

Low deprivation

Medium

High deprivation

I thought the purpose of the study was to encourage people to take part in different activities

$65-74$ years

$75-84$ years

Male

Female

Low deprivation

Medium

High deprivation

\section{B) Concerns About Privacy}

I do not want a research nurse coming to my home

$65-74$ years
$75-84$ years

Male

Female

Low deprivation

Medium

High deprivation

I do not want to be asked questions about my personal details/activities

$65-74$ years

$75-84$ years

Male

Female

Low deprivation

Medium

High deprivation
Yes (\%) No (\%) No box Selected (\%) $\quad \chi^{2} \quad$ P 
Table 4: Reasons for Refusal To Participate By Age, Gender and Deprivation (Continued)

\section{C) Personal Reasons}

I am too old to take part in a study

$65-74$ years

$75-84$ years

Male

Female

Low deprivation

Medium

High deprivation

I am not well enough to take part in the study

$65-74$ years

$75-84$ years

Male

Female

Low deprivation

Medium

High deprivation

D) Not Interested In The Research

I am not interested in a study on activities of older people

$65-74$ years
$75-84$ years
Male
Female
Low deprivation
Medium
High deprivation
I am not interested in research
$65-74$ years
$75-84$ years
Male
Female
Low deprivation
Medium
High deprivation

$\begin{array}{lllll}20 & 41 & 39 & 29.10 & <0.0001 \\ 52 & 21 & 27 & & \\ 40 & 33 & 28 & 2.17 & 0.338 \\ 36 & 28 & 36 & & \\ 38 & 27 & 35 & 0.88 & 0.927 \\ 38 & 32 & 30 & & \\ 36 & 33 & 32 & & \\ & & & & \\ 21 & 41 & 38 & 5.87 & 0.053 \\ 34 & 37 & 29 & & \\ 27 & 43 & 30 & 1.59 & 0.451 \\ 29 & 36 & 36 & & \\ 25 & 37 & 38 & 1.94 & 0.747 \\ 28 & 40 & 32 & & \\ 32 & 40 & 29 & & \end{array}$

\begin{tabular}{lllll}
35 & 33 & 32 & 1.64 & 0.441 \\
28 & 34 & 38 & & \\
34 & 37 & 29 & 3.14 & 0.209 \\
30 & 30 & 40 & & \\
28 & 33 & 39 & 2.99 & 0.559 \\
33 & 38 & 29 & & \\
34 & 29 & 37 & & \\
27 & 36 & & & \\
28 & 35 & 37 & 0.02 & 0.991 \\
33 & 38 & 37 & & \\
22 & 33 & 29 & 6.22 & 0.045 \\
17 & 33 & 45 & & \\
29 & 38 & 50 & 9.39 & 0.052 \\
36 & 36 & 34 & & \\
& & 29 & & \\
\hline
\end{tabular}


$[14,15]$, despite the fact that a range of theories of patient behaviour may contain relevant concepts that could be used in their design (e.g. self-efficacy, subjective norm etc) [29]. A Cochrane review of strategies to improve response rates in observational studies revealed a concentration on modes of administration [14,30]. Only two studies focussed on the information leaflets used in recruitment, and these only examined the appropriate level of detail rather than actual content per se. No strategies sought to address the beliefs or attitudes of potential participants towards participation or non-participation. Since this review a number of studies have targeted concerns and demonstrated that recruitment strategies that are designed to address these can have a significant impact [16,31-33]. One recent study demonstrated an increase in participation from $40 \%$ to $70 \%$ [34].

It is possible that there is so much heterogeneity between trial contexts that established behavioural theory would contribute little as reasons would be trial specific. Indeed despite identifying 15 studies in a Cochrane review of recruitment strategies the authors were uncertain of the generalisability of the finding to other trials as there was too much heterogeneity between studies to perform metaanalysis [15].

\section{The meaning of non-consent}

The information sheet for the main study which had been subject to careful design, consisted of neutral, non-coercive language and had been approved by the local research ethics committee (LREC). However, we found that many expressions of refusal were based on misunderstandings about the research and therefore fell short of being informed and comprehended non-consent.

Ethics committees attempt to ensure that when people decide to participate in a research study their consent is meaningful. This depends on three issues: that they are adequately informed, that they adequately comprehend, and finally that they are not coerced. However, LRECs and researchers do not routinely apply the same criteria to nonparticipation. Our data raises questions over the meaningfulness of the expressions of non-consent received in this study. Furthermore, it suggests that even carefully designed and LREC approved information provision can result in poorly comprehended information, undermining meaningful decisions about participation.

There are good reasons why we should seek to ensure that expressions of non-consent (as well as consent) are meaningful and valid. Firstly, it can be argued that potential participants have the right to be provided with full and accurate information prior to making a decision not to participate. Arguably this includes being informed of the consequences of their non-participation (and a low partic- ipation rate overall) on the usefulness of the research and for patient care. Just as we should seek to ensure that those who agree to participate do not subsequently regret their decision, so too we should seek to ensure that those who refuse do not look back and wish that they had taken part or been given more information. Since many people in our study appeared to have declined participation due to misunderstandings it is quite possible that a correction of these misunderstandings would lead to some regretting their earlier decision.

A second reason to pursue meaningful non-consent stems from the researchers' ethical obligations to those who do consent. If we do not actively explore the reasons behind and meaning of non-consent and develop solutions, we may be at risk of wasting the time and generosity of those members of the public who do agree to contribute. If consent was given that knowledge would be advanced then researchers must seek to ensure that this is achieved. Obtaining a sufficiently large and unbiased sample may be a prerequisite for this.

Ideally therefore, refusal to participate should reflect a fully comprehended and active evaluation of the pros and cons of participation If this is the case, then perhaps nonresponse or refusal to participate should be regarded as a behaviour that it is not only legitimate to explore and perhaps attempt to change, but one that it would be negligent or unethical not to. However, the challenges of pursuing such research should not be overlooked. The acceptability and ethical implications of studies designed to explore non-participation has been largely omitted from debates around recruitment despite some using arguably intrusive methods. One such study [35] conducted personal visits to the homes of those who had previously written to refuse participation. This approach yielded a final 93\% participation rate. Others have only conducted home visits to those who did not respond to an initial request to participate $[10,36,37]$. Telephone and postal follow-up of all non-responders (including those who refused to participate) have also frequently been used[3,38]. It is interesting that none of these papers mention ethical issues.

\section{The challenges posed by "opt-in"}

We used an "opt-out" approach to recruitment that is increasingly being rejected by research ethics committees in favour of "opt-in" approaches [39]. The vast majority $(81 \%)$ of those who did not wish to participate in the main survey returned a postcard as part of the opt-out. This would suggest that an active decision against participation had been made. Few studies among older people distinguish between positive opt out and passive non-participation, although two studies involving psychiatric interviews $[4,40]$ also found a high positive opt-out rate. However one of these [40] made up to ten attempts to 
contact subjects, which may have increased the likelihood that subjects actively opted out. Another study, a trial of influenza vaccination, reported that $50 \%$ of those not taking part had also positively opted out [37].

If researchers wish to maintain or increase response rates to research and ensure that consent and non-consent is informed and meaningful then an understanding of the issues that concern potential participants is crucial [1], as is a knowledge of concepts that are known to influence behaviour more generally [41]. However, the increasing requirement to use opt-in procedures may undermine such attempts. Under the opt-out procedure recruitment depends largely on the inertia of individuals [39]. However, the move towards an "opt-in" system has now reversed this. The failure to act leads to non-inclusion, but also means that non-inclusion is less likely to stem from any meaningful consideration of the pros and cons. Consequently, "non-participation" may not be an intentional or volitional act reflecting an unwillingness to take part but rather reflect a lack of intention or volition altogether, particularly among individuals who are less inclined to be actively involved in health-related decision making but instead leave it to professionals. Indeed a recent randomised trial has found evidence to support this [2]. Nonparticipation may therefore sometimes stem less from a cognitive process and more from an adopted and established role in relation to health services generally. Furthermore, if data suggests that some individuals may have a preference not to be involved in some decisions, this might support Parker's recent argument for the use of data without consent for some low-risk research: "Patients may have good reasons to expect, or come to expect, that their records will be used without their consent for some low risk research, under certain conditions. Where this is the case, such expectations provide reasonable grounds for considering such research to be ethical."[42]p183.

\section{Conclusion}

Findings from this study have potential implications for recruitment and consenting practices when seen against the context of recent changes to ethical requirements and data protection. Firstly, the design of information leaflets and the verbal consenting approach should not be excluded from the concept of evidence-based practice. If there is empirical evidence or relevant behavioural theory to suggest the best means to achieve meaningful consent then these should be pursued. Secondly, expressions of non-consent need to be subject to further research and not seen as out of bounds as a focus for investigation. A limited amount of additional research may be important to ensure we are conducting our research recruitment to a high standard.
Thirdly, in pursuing the ideal of meaningful consent researchers could consider presenting potential participants with two options: participation and non-participation and highlight the pros and cons of each in a manner not dissimilar to that of two treatment options. At present, it could be argued that the information insisted upon by ethics committees focuses on that needed to take part rather than that needed to decide not to take part. The consequences of non-participation for example (for both the individual, science, and the public), are rarely conveyed, probably through fear of being coercive. While recent commentators have questioned whether we currently know what information potential participants need in order to participate in a research project it may also be worth considering what information they may require in order to know whether they should refuse to participate [1]. The presentation of such information may contribute to ensuring that both consent and non-consent are meaningful.

\section{Competing interests}

The author(s) declare that they have no competing interests.

\section{Authors' contributions}

IKC and LI conceived the idea for the study. All authors were involved in the design. AM collected the data. LI and IKC analysed the data. BW wrote the paper. All authors edited, revised and approved the final manuscript.

\section{Acknowledgements}

We are grateful to the many GPs in Dundee who gave their support to this study. This study was funded by the Chief Scientist Office, Scottish Executive Health Department: grant application number $\mathrm{CZH} / 4 / 5$. The design, conduct and analysis of this study was completely independent of the funders.

\section{References}

I. Hewison J, Haines A: Overcoming barriers to recruitment in health research. British Medical Journal 2006, 333:300-302.

2. Trevena $L$, Irwig $L$, Barratt A: Impact of privacy legislation on the number and characteristics of people who are recruited for research: a randomised trial. Journal of Medical Ethics 2006, 32:473-477.

3. Locker D, Slade GD, Leake JL: The response rate problem in oral health surveys of older adults in Ontario. Can J Public Health I990 May-Jun;8 I (3):2 I 0-4 1990, 8 I:2 I 0-2I 4.

4. Launer LJ, Wind AW, Deeg DJH: Nonresponse Pattern and Bias in a Community-based Cross-sectional Study of Cognitive Functioning among the Elderly. American Journal of Epidemiology 1994, 139:803-812.

5. Hoeymans N, Feskens EJ, Van Den Bos GA, Kromhout D: Nonresponse bias in a study of cardiovascular diseases, functional status and self-rated health among elderly men. Age Ageing 1998, 27:35-40.

6. Herzog AR, Rodgers WL: Age and response rates to interview sample surveys. J Gerontol 1988, 43:S200-5.

7. Boersma F, Eefsting JA, Van Den Brink W, Van Tilburg W: Characteristics of Non-Responders and the Impact of NonResponse on Prevalance Estimates of Dementia. International Journal of Epidemiology 1997, 26:1055-1062. 
8. van den Akker M, Buntinx F, Metsemakers JF, Knottnerus JA: Morbidity in responders and non-responders in a register-based population survey. Fam Pract 1998, 15:26 I-263.

9. Carter WB, Elward K, Malmgren J, Martin ML, Larson E: Participation of older adults in health programs and research: a critical review of the literature. Gerontologist I991, 31:584-592.

10. Freudenstein U, Arthur AJ, Matthews RJ, jagger C: Community surveys of late-life depression: who are the non-responders? Age and Ageing 200I, 30:5I7-52I.

II. Smeeth L, Fletcher AE, Stirling S, Nunes M, Breeze E, Ng E, Bulpitt CJ, Jones $D$ : Randomised comparison of three methods of administering a screening questionnaire to elderly people: findings from the MRC trial of the assessment and management of older people in the community. BMJ 200I, 323:1403-I407.

12. Halbert JA, Silagy CA, finucane P, Withers RT, Hamdorf PA: Recruitment of Older Adults for a Randomized, Controlled Trial of Exercise Advice in a General Practice Setting. J Am Geriatr Soc 1999, 47:477-481.

13. Ory MG, Lipman PD, Karlen PL, Gerety MB, Stevens VJ, Singh MAF, Buchner DM, Schechtman KB, FICSIT Group: Recruitment of Older Participants in Frailty/Injury Prevention Studies. Prevention Science 2002, 3:1-22.

14. Roberts EP, Clarke M, DiGuiseppi C, Pratap S, Wentz R, Kwan I, Cooper $R$ : Methods to increase response rates to postal questionnaires. The Cochrane Database of Methodology Reviews 2003.

15. Mapstone J, Elbourne D, Roberts I: Strategies to improve recruitment to research studies. 2002, The Cochrane Database of Methodology:

16. Mills N, Donovan JL, Smith M, Jacoby A, Neal DE, Hamdy FC: Perceptions of equipoise are crucial to trial participation; a qualitative study of men in the ProTec study. Controlled Clinical Trials 2003, 24:272-282.

17. N K Aaronson, E Visser-Pol, G H M W Leenhouts, MJ Muller, A C M S, F S A M D, R B Keus, C C E Koning, W W BH, J A D, Dubbelman $R$ : Telephone-based nursing intervention improves the effectiveness of the informed consent process in cancer clinical trials. Journal of Clinical Oncology 1996, 14:984-996.

18. Lowton $\mathrm{K}$ : Trials and tribulations: understanding motivations for clinical research participation amongst adults with cystic fibrosis. Social Science \& Medicine 2005, 61:1854-1865.

19. Barrett PH, Beck A, Schmid K, Fireman B, Brown JB: Treatment decisions about lumbar herniated disk in a shared decisionmaking program. It Comm J Qual Improv 2002, 28:2 I I-2 I9.

20. Fry C, Dwyer R: For love or money? An exploratory study of why injecting drug users participate in research. Addiction 2001, 96:1319-1325.

21. Liaschenko J, Underwood SM: Children in research: Fathers in cancer research - meanings and reasons for participation. Journal of Family Nursing 200I, 7:7I-91.

22. Al-Shahi R, Vousden C, Warlow C: Bias from requiring explicit consent from all participants in observational research: prospective, population based study. British Medical Journal 2005, 33 I:942-946.

23. Junghans C, Feder G, Hemmingway H, Timmis A, Jones M: Recruiting patients to medical research: double blind randomised trial of "opt-in" versus "opt-out" strategies. British Medical Journal 2005, 331:940-943.

24. Crombie I, Irvine L, Williams B, McGinnis A, Slane P, Alder E, McMurdo M: Why older people do not participate in leisure time physical activity. Age and Ageing 2004, 33:287-292.

25. Carstairs $V$, Morris R: Deprivation and Health in Scotland. Aberdeen, Aberdeen University Press; 1991.

26. McLoone P: Cartairs Scores for Scottish Postcode Sectors from the 199I Census. Glasgow, Public Health Research Unit; 1991 .

27. Dobson AJ: An Introduction to Generalised Linear Models. First edition. London, Chapman Hall; 1990.

28. Kelsey JL, O'Brien LA, Grisso JA, Hoffman S: Issues in carrying out epidemiologic research in the elderly. $\mathrm{Am} /$ Epidemiol 1989 I30:857-866.

29. Conner M, Sparks P: The Theory of Planned behaviour and Health behaviours. In Predicting Health Behaviour Edited by: Conner M and Norman P. Buckingham, Open University Press; 1998: I2I-162.

30. Edwards P, Roberts I, Clarke M, DiGuiseppi C, Pratap S, Wentz R, Kwan I, Cooper R: Methods to increase response rates to postal questionnaires (Review). Volume Issue no.4. , The Cochrane Database of Methodology Reviews; 2003.

31. Donovan JL, Peters TJ, Noble S, Powell P, Gillatt D, Oliver SE, Lane JA, Neal DE, Hamdy DFC: Who can best recruit to randomized trials? Randomized trial comparing surgeons and nurses recruiting patients to a trial of treatments for localized prostate cancer (the ProTec study). Journal of Clinical Epidemiology 2003, 56:605-609.

32. Fleissig A, Jenkins V, Fallowfield L: Results of an intervention study to improve communication about randomised clinical trials of cancer therapy. European Journal of Cancer 200I, 37:322-331.

33. Jenkins V, Leach L, Fallowfield L, Nicholls K, Newsham A: Describing randomisation: patients' and public's preferences compared with clinicians' practice. British Journal of Cancer 2002, 87:854-858.

34. Donovan J, Mills N, Smith M, Brindle L, Jacoby A, Peters T, Frankel S, Neal D, Hamdy F: Improving design and conduct of randomised trials by embedding them in qualitative research: ProtecT (prostate testing for cancer and treatment) study. British Medical Journal 2002, 325:766-770.

35. Norton MC, Breitner JC, Welsh KA, Wyse BW: Characteristics of nonresponders in a community survey of the elderly. J Am Geriatr Soc 1994, 42: 1 252-1256.

36. McDonnell H, Long AF, Harrison BJ, Oldman C: A study of persons aged 65 and over in the Leeds Metropolitan District. J Epidemiol Community Health 1979, 33:203-209.

37. Allsup SJ, Gosney MA: Difficulties of recruitment for a randomized controlled trial involving influenza vaccination in healthy older people. Gerontology 2002, 48: I70-173.

38. Rupp I, Triemstra M, Boshuizen HC, Jacobi CE, Dinant HJ, van den Bos GA: Selection bias due to non-response in a health survey among patients with rheumatoid arthritis. Eur J Public Health 2002, I 2: I3I-135.

39. Sommerville A: Commentary: What's wrong with opting out? British Medical Journal 200I, 322:

40. Heun R, Hardt J, Muller H, Maier W: Selection bias during recruitment of elderly subjects from the general population for psychiatric interviews. Eur Arch Psychiatry Clin Neurosci 1997, 247:87-92.

4l. Ajzen I: From intentions to action: a theory of planned behaviour. In Action Control: From Cognitions to Behaviours Edited by: Kuhl J and Beckman J. New York, Springer; I 985: I I-39.

42. Parker M: When is research on patient records without consent ethical? Journal of Health Services Research \& Policy 2005, 10:183-186

\section{Pre-publication history}

The pre-publication history for this paper can be accessed here:

http://www.biomedcentral.com/1472-6963/7/59/prepub

Publish with Bio Med Central and every scientist can read your work free of charge

"BioMed Central will be the most significant development for disseminating the results of biomedical research in our lifetime. "

Sir Paul Nurse, Cancer Research UK

Your research papers will be:

- available free of charge to the entire biomedical community

- peer reviewed and published immediately upon acceptance

- cited in PubMed and archived on PubMed Central

- yours - you keep the copyright
BioMedcentral 